\title{
LOW VELOCITY INELASTIC COLLISIONS
}

\author{
By Clarence Zener* \\ California Institute of Technology \\ Pasadena, California.
}

(Received June 1, 1931)

Abstract

The probability that a low velocity collision be inelastic is shown to depend in a relatively simple manner upon three factors: the magnitude of the change in total internal energy, $\Delta E$; the matrix element, with respect to the initial and final states, of the interaction energy at the closest distance of approach, $W_{12}$; and the "time of collision," $\tau$, a function of the relative velocity and of the sharpness of the collision. An approximation to this probability is

$$
P=\left[\frac{2 \pi W_{12}}{h} \tau\left(!+\epsilon_{1} \beta^{\epsilon_{2}}\right) e^{-\epsilon_{3} \beta}\right]^{2}
$$

where $\beta=2 \pi \tau \Delta E / h$ and $\epsilon_{1}, \epsilon_{2}, \epsilon_{3}$ are nearly unity, their precise values depending upon the characteristics of the particular collision.

\section{INTRODUCTION}

$T^{H}$ $\mathrm{HE}$ Born method of treating a system involving a perturbation dependent on the time has frequently been applied with success in the study of high velocity collisions. Similar success has not been met in low velocity collisions. This failure is due in part to the reluctance of modern theoretical physicists to work with classical methods even when their use is justifiable. This is illustrated by the problem of the transfer of energy between atoms. If the atoms are replaced by sets of linear oscillators, as in the dispersion theory, the classical equations give results qualitatively better than the approximate quantum mechanical treatment of Kallman and London, ${ }^{1}$ and , in the most interesting cases, identical with the more exact quantum mechanical results of Rice. ${ }^{2}$ Again, the author ${ }^{3}$ has shown how to calculate the transfer of vibrational energy between molecules. His wave equations were so complex that in order to obtain numerical results crude approximations had to be made. With the introduction of classical concepts these approximations are unnecessary.

The major difficulty in a precise quantum mechanical treatment, as given by Rice and the author, occurs in operations involving the relative coordinates of the centers of gravity of the two colliding systems. But the centers of gravity may be described approximately by classical equations. In this paper it is shown how a large class of low velocity inelastic collisions may be readily treated starting from that wave equation of the internal coordinates in which the relative coordinates occur as parameters and depend upon time

* National Research Fellow

${ }^{1}$ Kallman and London, Zeits. f. physik. Chem B. 2, 207 (1929).

2 O. K. Rice, Proc. Nat. Acad. 17, 34 (1931).

${ }^{3}$ C. Zener, Phy. Rev. 37, 556 (1931). 
in a manner determined by Newtonian equations. In these Newtonian equations the potential is taken to be the exact interaction potential averaged over all the internal coordinates.

\section{General Theory}

Let $H_{0}$ be the unperturbed Hamiltonian of the internal coordinates of the two systems, $\psi_{n}$ and $E_{n}$ the corresponding eigenfunctions and eigenwerte. The subscript refers to the total aggregate of internal quantum numbers. The mutual interaction $V$ is appreciably different from zero only during a finite interval of time. Hence the transition probability, obtained by the variation of constants method, approaches a constant value at infinite time. This limiting value, representing the probability of a transition during a collision in which $n^{\prime} \rightarrow n^{\prime \prime}$ is

$$
P=\left.\left|\frac{2 \pi}{h} \int_{-\infty}^{\infty} V^{\prime \prime}\{X(t)\} e^{2 \pi i t \Delta E / h} d t\right|\right|^{2} .
$$

Here $V^{\prime \prime}(X)=\int \psi_{n^{\prime \prime}}{ }^{*} V \psi_{n^{\prime}} d v$, and $\Delta E=\left|E_{n^{\prime}}-E_{n^{\prime \prime}}\right|$. Let $E$ be the constant total energy of the system. Then the relative coordinates $X$ are to be obtained as functions of time by solving the classical equation of motion whose potential energy and total energy are $\left(V_{n^{\prime}}{ }^{\prime}+V_{n^{\prime \prime}} n^{\prime \prime}\right) / 2$ and $E-\left(E_{n^{\prime}}+E_{n^{\prime \prime}}\right) / 2$ respectively. The arbitrariness of this determination of the relative coordinates increases with the differences $\left|V_{n^{\prime}} n^{\prime}-V_{n^{\prime}}, n^{\prime \prime}\right|$ and $\left|E_{n^{\prime}}-E_{n^{\prime \prime}}\right|$, so that this method becomes inapplicable when these differences are large.

Let the origin of time be chosen at the moment of closest approach, so $V^{\prime \prime}(t)$ has the maximum value $W_{12}=V^{\prime \prime}(0)$. With each inelastic collision we may associate a time of collision defined as that time interval $\tau$ whose product with $W_{12}$ is equal to the integrated value of $V^{\prime \prime \prime}(t)$, i.e. $W_{12} \tau=\int_{-\infty}^{\infty} V^{\prime \prime}(t) d t$. We may then write

where

$$
P=\left|\frac{2 \pi W_{12}}{h} \tau S\right|^{2}
$$

$$
S=\frac{1}{W_{12} \tau} \int_{-\infty}^{\infty} V_{1}^{\prime \prime}(t) e^{2 \pi i t \Delta E / h} d t
$$

We see that $S$ has the maximum value of unity when $\Delta E=0$, and that it decreases rapidly as $2 \pi \Delta E \tau / h$ becomes large. Before examining in detail the dependence of $S$ upon $\Delta E$, it will be interesting to review the classical concepts of energy interchange.

We consider the energy interchange between two linear oscillators $A, B$, with angular frequencies $w_{1}$, $w_{2}$. Let only $B$ be initially oscillating, and let the coupling be suddenly introduced. $A$ will start oscillating with a phase lag with respect to $B$. If $w_{1}=w_{2}$, this phase difference will remain constant until all the energy of $B$ has been given to $A$. The situation is then reversed, $B$ will start oscillating with a phase lag, and gradually regain its initial energy. However, if $w_{1} \neq w_{2}$, the phase differences will be continually changing. $B$ will start lagging in phase before all its energy has been lost, and hence $A$ will 
never acquire all the energy of $B$. The maximum energy of $A$ will decrease with increasing $\left|w_{1}-w_{2}\right|$. This maximum energy will also depend upon the strength of the coupling. Hence if this coupling changes from zero to a maximum and then to zero so gradually that its change during the time interval $1 /\left|w_{1}-w_{2}\right|$ vanishes, the total energy transferred to $A$ approaches zero. Thus increasing $\left|w_{1}-w_{2}\right|$ has the double effect of reducing the maximum energy of $A$ and of reducing the ratio of the final energy of $A$ to this maximum energy. To obtain a quantitative idea of these effects in actual collisions, we shall consider $B$ to move past $A$ with a constant velocity $v$ and a closest distance of approach $p$. The coordinates of the oscillators $A, B$ namely $\xi, \eta$ are taken to be normal to the plane of $p$ and $v$. Then the interaction energy $e^{2} \xi \eta\left(p^{2}+v^{2} \xi^{2}\right)^{-3 / 2}$ gives rise to a transfer of energy from $B$ to $A$ of magnitude ${ }^{4}$

$$
\Delta w=\frac{1}{2 m}\left[\frac{e^{2} \eta_{0}}{v p^{2}} \beta K_{1}(\beta)\right]^{2} \simeq \frac{1}{2 m}\left[\frac{e^{2} \eta_{0}}{v p^{2}}\left(1+\beta^{1 / 2}\right) e^{-\beta}\right]^{2}
$$

provided we may consider $B$ as undisturbed by $A$. Here $\beta=(P / v)\left|w_{1}-w_{2}\right|, \eta_{0}$ is the amplitude of $\eta$, and $m$ is the mass associated with $\xi$. If we now translate these classical results into quantum language by defining $P=2 \pi \Delta W / h w_{1}$ as the probability that $A$ become excited, and $W_{12}=e^{2} \xi^{1} \eta_{0} / p^{3}$ where $\xi_{0}^{1}$ is the matrix element of $\xi$ over the normal and first excited states we obtain

$$
P \simeq\left[\frac{2 \pi W_{12}}{h} \tau\left(1+\beta^{1 / 2}\right) e^{-\beta}\right]^{2}
$$

where now $\tau=p / v, \beta=\tau\left|w_{1}-w_{2}\right|=2 \pi \tau \Delta E / h$. Here we have used the quantum result that $4 \pi m\left(\xi_{0}\right)^{2} w_{1} / h=1$. It is interesting to note that a formula identical to this is obtained from the quantum mechanics, as we should expect from the dispersion theory.

As a function of $\tau, P$ has the maximum value $\left(2 W_{12} / \Delta E\right)^{2}$ when $\tau=\tau_{0} \equiv h(2 \pi \Delta E)^{-1}$. In the classical picture, when $\tau=\tau_{0}$ the oscillators are effectively separated just before $A$ begins to return energy to $B$. As $\tau$ becomes much smaller than $\tau_{0}$, the systems do not remain in contact long enough for much energy to be transferred. When $\tau \gg \tau_{0}$, energy fluctuates many times between $A$ and $B$, the maximum energy of $A$ gradually decreasing as the systems are separated.

\section{Applications}

Inelastic low velocity collisions in actual experiments are too complex to solve in a straightforward manner by quantum mechanics. We must replace these collisions by simpler collisions in which irrelevant elements are omitted. But in deciding which elements are important we must use our physical intuition which is based on classical mechanics. In the previous section we found that the classical mechanics, supplemented by the quantum conditions of dispersion theory, gives results identical to the quantum mechanics in the simple collision of two linear oscillators. Moreover, we were able to interpret the

4 This may be obtained by a method analogous to that of N. Bohr, Phil. Mag. 25, 10 (1913). 
results in terms of elementary concepts. Hence if we are able to express the probability of an inelastic collision in a form similar to that for two linear oscillators, say as

$$
\begin{aligned}
& P=\left[\frac{2 \pi W_{12}}{h} \tau\left(1+\epsilon_{1} \beta^{\epsilon_{2}}\right) e^{-\epsilon_{3} \beta}\right]^{2} \\
& \beta=\frac{2 \pi \Delta E}{h} \tau
\end{aligned}
$$

we shall be able to form a physical picture of the collision, and thus see why only certain factors are important.

The efficiency of an inelastic collision is usually expressed by an effective cross section, $\sigma_{\text {eff. }}$ Let $r_{E}$ be the closest distance of approach in a head-on collision whose relative kinetic energy is $E$. Then if $\sigma_{\text {eff }}>\pi r_{E}{ }^{2}$, an approximation may be obtained by the method of Rice. ${ }^{2}$ That closest distance of approach $p_{0}$ is found for which the transition probability is appreciable, say $\frac{1}{2}$. Then $\sigma_{\text {eff }} \cong \pi p_{0}{ }^{2}$. This is justified since the transition probability diminishes rapidly when the closest distance of approach becomes larger than $p_{0}$. If $\sigma_{\text {eff }} \ll \pi p_{0}{ }^{2}$, an upper limit may readily be found by calculating the transition probability $P$ for a head-on collision. Then $\sigma_{\text {cff }}=\lambda P \pi r_{E}{ }^{2}, \lambda \leqq 1$. The precise value of $\lambda$ varies with different collisions, ranging from 0.1 to 0.5 for those collisions which have been examined.

When the effective cross section $\sigma_{\text {eff }}$ is larger than $\pi r_{E}{ }^{2}$, we need consider only those collisions whose closest distance of approach $p$ is so large that the centers of gravity of the two systems may be taken to move in straight lines. If $p$ is so large that the interaction between two atoms is essentially that of dipoles, the probability of an energy transfer between an excited and normal atom is found from (1) to be approximately

$$
\begin{aligned}
& P=\left[\frac{2 \pi W_{12}}{h} \tau\left(1+4 \beta^{3 / 2}\right) e^{-\beta}\right]^{2} \\
& \beta=\frac{2 \pi \Delta E}{h} \tau .
\end{aligned}
$$

Here $W_{12}=e^{2} / 2 p^{3}\left(x_{1} \pm i y_{1}\right){ }^{\prime \prime}\left(x_{2} \pm i y_{2}\right){ }^{\prime \prime}, \tau=p / v$, and $\Delta E$ as usual refers to the magnitude of the change in the total internal energy. The formula of Kallman and London ${ }^{1}$ is essentially obtained as the special case when $v$ is so adjusted that $\beta=1$, Hence their $\sigma_{\text {eff }}$ decreases too slowly with increasing $\Delta E$. When $\Delta E$ is small in comparison to the relative kinetic energy of translation, and when $\beta>1,(2)$ is identical to the formula of Rice. ${ }^{2}$ Rice's formula is less general than (2), not being defined for $0<\beta<1$. Substitution of numerical values shows that at room temperature unless $\Delta E<0.01$ volt, $P$ is very small when $p \geqq 5 \times 10^{-8} \mathrm{~cm}$. Hence the assumptions leading to (2) are rarely justified.

Since for nearly all inelastic collisions, $\sigma_{\text {eff }} \ll \pi r_{E}^{2}$, in most cases it will be sufficient to find $P$ only for head-on collisions, and then set $\sigma_{\text {eff }}<P \pi r_{E}{ }^{2}$. 
In a head-on collision the only relative coordinate is $r$, the distance between the centers of gravity of the two systems. The interaction integrals $V,{ }^{\prime \prime}$ depend upon $r$ approximately as

$$
V^{\prime \prime}=W_{12} e^{-\alpha\left(r-r_{E}\right)},
$$

where $r_{E}$ is the closest distance of approach when the relative kinetic energy is $E$. The approximate dependence of $r$ upon time is found by solving the classical equation of motion in which the potential is $E e^{-\alpha\left(r-r_{E}\right)} .^{5}$ This solution is $r-r_{E}=(1 / \alpha) \log \{(1+\cosh \alpha v t) / 2\}$. The velocity $v$ is defined by $\frac{1}{2} m v^{2}=E, m$ being the reduced mass of the system. Substitution into (1) gives

$$
P=\left[\frac{2 \pi W_{12}}{h} \int_{-\infty}^{\infty} \frac{2 e^{2 \pi i t \Delta E / h}}{1+\cosh \alpha v t} d t\right]^{2}
$$

Integration gives, defining $\tau=4(\alpha v)^{-1}$,

$$
\begin{aligned}
P & =\left[\frac{2 \pi W_{12}}{h} \tau \frac{\beta}{\sinh \beta}\right]^{2} \\
& \cong\left[\frac{2 \pi W_{12}}{h} \tau(1+2 \beta) e^{-\beta}\right]^{2} \\
\beta & =\frac{2 \pi \Delta E}{h} \cdot \frac{\pi \tau}{4} .
\end{aligned}
$$

Eqs. (4) may be applied to that large group of collisions in which the interaction energy may be written as (3). We see that the important factors of a low velocity inelastic collision are the relative velocity, the sharpness of the interaction potential, which together determine $\tau$, the energy resonance $\Delta E$, and the maximum value of the matrix element of the interaction energy, $W_{12}$. The precise form of the interaction potential is thus not very important. With a knowledge of these three quantities we may at once obtain the magnitude of $\sigma_{\text {eff. }}$.

As a numerical example, let $P$ be the probability that in a head-on collision at room temperature an excited diatomic molecule in its first vibrational state transfer its energy of excitation to a normal diatomic molecule. Here typical values are $W_{12} \cong 0.0005$ volts, $\tau=2 \times 10^{-13}$ secs, and $\Delta E=0.02$ volts, which on substitution in Eq. (4) leads to $\beta=6$ and $P=2 \times 10^{-5}$.

${ }^{5}$ For a justification of this potential, see ref. 3 , also London, Zeits. f. physik. Chem. B. 11, 237 (1930), and Kirkwood, Phys. Rev. 37, 835 (1931) 